\title{
Les associations dans le processus de rationalisation des données naturalistes
}

\author{
Pierre Alphandéry, Agnès Fortier \\ Sociologues, INRA, UMR1048 SADAPT, 94205 Ivry-sur-Seine, France
}

\begin{abstract}
Rien ne vaut un récit socio-anthropologique de la construction des politiques publiques d'environnement pour rendre intelligibles aux lecteurs de NSS les rapports qui s'établissent entre les différents types de savoirs sur la nature. D'un côté, les «associations naturalistes", où scientifiques et amateurs sont des acteurs indispensables pour l'observation de la nature, la collecte et la production des données. De l'autre, un système d'information pour la nature et les paysages (SINP) mis en place par les pouvoirs publics pour établir une normalisation des données et en faire la base cognitive du cadrage de son action. Confrontation de deux mondes qui interpelle la nature des connaissances produites et affecte les identités, en particulier dans les associations qui, pour certaines d'entre elles, se sentent instrumentalisées.
\end{abstract}

La Rédaction

\section{Mots-clés :}

action publique ;

biodiversité ; données naturalistes ; rationalisation ; associations naturalistes

\begin{abstract}
Résumé - L'action publique en faveur de la préservation du patrimoine naturel et de la conservation de la biodiversité a conduit à développer et à rationaliser la production des connaissances sur la nature. La mise en œuvre par le ministère de l'Écologie, du Développement et de l'Aménagement durables du système d'information sur la nature et les paysages (SINP), destiné à normaliser le recueil des données, est censée remédier à leur caractère lacunaire et faciliter leur mobilisation. Les associations naturalistes figurant parmi les principaux pourvoyeurs d'informations, nous questionnerons les conséquences du recours à ce dispositif sur leur fonctionnement. L'approche socio-anthropologique développée conduit à appréhender la production des données à la fois comme produit d'un dispositif global de normalisation et comme le fruit d'une activité associative, et ainsi de mettre en perspective la logique du SINP avec la logique associative.
\end{abstract}

\section{Keywords: public action; biodiversity conservation; naturalist data; rationalization; NGOs}

\begin{abstract}
NGOs in the process of rationalization of naturalist data. The various French public actions in support of biodiversity conservation have led to developing and rationalizing the production of knowledge on nature. Implementation of the "système d'information sur la nature et les paysages" (SINP) by the Ministry of Ecology aims to standardize data recording. Its purpose is to remedy the lacks in data recording and make data retrieval easier. Our paper focuses on the consequences of SINP implementation for NGOs who are the main data collecting structures. Our analysis is based on a socio-anthropological approach and considers the production of data to be linked to a general process of standardization on the one hand and as result of NGO activity on the other. We question whether the two logics are compatible.
\end{abstract}

Depuis plusieurs décennies, l'action publique pour la protection du patrimoine naturel et la conservation de la biodiversité s'appuie de plus en plus sur la production de données naturalistes ${ }^{1}$. Issues en France d'un processus de «mise en forme » d'observations opérées sur le terrain par des amateurs et des professionnels, elles constituent un outil essentiel de la connaissance et de la gestion de

Auteur correspondant : A. Fortier, fortier@ivry.inra.fr

${ }^{1}$ Cet article est issu d'une communication présentée lors du colloque Instituer le développement durable, organisé par la Faculté des sciences juridiques, politiques et sociales de Lille 2, du 8 au 10 novembre 2007. la nature. Ainsi, la Stratégie nationale pour la biodiversité présente comme un «impératif » de «mettre en place un dispositif d'observation opérationnel qui fournisse des données fiables, objectives et représentatives de l'état de la biodiversité, pour le pilotage de la stratégie et de ses plans d'action ainsi que pour l'information de la société » (Ministère de l'Écologie et du Développement durable, 2004, p. 39).

La volonté de pallier le caractère lacunaire, hétérogène, controversé, de la collecte des données naturalistes et de la rendre plus efficace n'est pas nouvelle. Elle s'est manifestée depuis trente ans par un processus 
constant de rationalisation. Cependant, le dispositif ${ }^{2}$ mis en place, en 2007, à l'instigation du ministère de l'Écologie, du Développement et de l'Aménagement durables (MEDAD) - dénommé système d'information pour la nature et les paysages (SINP) - apparaît d'une ambition inégalée, puisqu'il cherche à définir des normes communes encadrant le recueil des données sur les espèces, les habitats et les paysages (Encadré 1$)^{3}$. Si les données disponibles au sein de l'Hexagone sont produites par de nombreuses institutions publiques (universités, centres de recherche, établissements publics) et privées (bureaux d'études, etc.), les associations naturalistes, composées en majorité d'amateurs et de bénévoles, figurent parmi les principaux pourvoyeurs d'informations sur la nature. Cette particularité, que l'on ne rencontre pas dans d'autres domaines de l'environnement, comme celui de l'eau ou des risques, dotés de moyens plus conséquents, nous est apparue intéressante à interroger au regard du dispositif adopté par le ministère. D'autant plus que, depuis la loi sur la protection de la nature de 1976, ces associations ont été étroitement impliquées dans les mécanismes de l'action publique (Lascoumes, 1994). Elles ont ainsi dû faire valoir leurs connaissances et légitimer leurs compétences dans leurs domaines d'action. Le rôle des amateurs dans la production de données naturalistes alimente depuis quelques années la réflexion de ceux qui se réclament des sciences citoyennes (Charvolin, 2009). Notre questionnement déborde cette problématique, car il porte sur les effets du processus de rationalisation de la production des données au sein du milieu associatif. Le SINP est-il compatible avec la logique associative basée sur le bénévolat? Les procédures de standardisation et la transparence imposées dans ce nouveau cadre ne risquent-elles pas d'exclure les bénévoles et, avec eux, leurs données? Cela nous a conduit à appréhender la production des données naturalistes dans une double perspective : à la fois comme le résultat d'un dispositif de normalisation et comme le fruit d'une activité associative. Cette approche de type socio-anthropologique consiste, d'une part, en un travail de suivi du processus de rationalisation de la production des données naturalistes, dont l'aboutissement est la mise en œuvre du dispositif SINP ; d'autre part, elle s'appuie sur une analyse de type ethnographique destinée à rendre compte des modalités de production des connaissances, laquelle suppose de comprendre le fonctionnement des structures associatives.

\footnotetext{
2 Nous définissons la notion de dispositif comme un réseau tourné vers l'action, composé d'objets hétérogènes en interrelation, comportant des humains et des non-humains, des éléments matériels et discursifs (Boussard et Maugeri, 2003, p. 3).

3 Le protocole du SINP est présenté en annexe de la circulaire du ministre d'État, ministre de l'Écologie, du Développement et de l'Aménagement durables du 11 juin 2007, publiée dans le Bulletin officiel du 30 août 2007 (document en ligne : http:// www.naturefrance.fr/spip.php?article189).
}

Encadré 1. Le système d'information sur la nature et les paysages (SINP)

L'élaboration du SINP intervient à la suite d'une série d'initiatives portées à l'échelon régional par le monde associatif (Office des données naturalistes d'Alsace [ODONAT], Réseaux des acteurs de l'information naturaliste [RAIN] en Nord-Pasde-Calais). Elle s'inscrit en outre dans un cadre élargi qui reconnaît désormais comme un impératif la mise en place d'instruments de mesures, d'indicateurs, et leur coordination à l'échelle internationale en vue d'atteindre l'objectif affiché par la Convention sur la diversité biologique (CDB) de réduire la perte de biodiversité d'ici 2010 (Balmfort et al., 2005; Scholes et al., 2008). Le but principal du SINP consiste à rendre plus efficaces la collecte, le traitement et la mobilisation de l'information en définissant des outils et des normes collectives destinées à standardiser les données sur les espèces, les habitats naturels et les paysages. Selon un chargé de mission du ministère en charge de l'environnement, il « n'y a pas en France de tradition de travail en commun entre organismes. Chacun a monté sa banque de données, son observatoire ou ses réseaux. Le basculement des années 2000 tient à la prise de conscience que l'on ne peut continuer sans coordination, car cela empêche de valoriser les données » (entretien, septembre 2006). Le dispositif adopté est conçu comme « une organisation collaborative favorisant une synergie entre les acteurs pour la production, la gestion, le traitement, la valorisation et la diffusion des données sur la nature et les paysages. Pour faciliter la participation et l'interaction de tous les acteurs, aussi bien nationaux que locaux, l'organisation repose sur un niveau national et un niveau régional. La cohérence globale de cette organisation est fondée par des règles communes qui doivent être respectées par tous les acteurs » (protocole du SINP, juin 2007, cf. note 3).

Ainsi, la mise en œuvre du SINP traduirait, selon nous, le passage d'un mode de production des données fondé sur des rapports personnalisés, tels qu'ils prévalent au sein de la nébuleuse associative, à une logique impersonnelle et standardisée incarnée par le SINP.

La démarche qualitative fondée sur un travail d'observation participante s'est également appuyée sur une série d'entretiens conduits auprès de divers acteurs du monde associatif et de responsables nationaux et locaux impliqués dans des projets d'organisation des données naturalistes, avec le souci constant d'articuler différents niveaux d'analyse. Depuis les échelons locaux ${ }^{4}$ et régionaux, qui constituent des maillons essentiels dans la production des connaissances sur la nature et la mise en œuvre des dispositifs d'actions publics, jusqu'aux niveaux national, européen et international. Cette approche élargie de la question des données naturalistes et de la réalité associative a été rendue possible par les acquis issus de travaux antérieurs portant notamment sur la généalogie de l'écologisme (Alphandéry et al., 1991) et sur

\footnotetext{
${ }^{4}$ Les enquêtes de terrain ont été conduites principalement et par ordre d'importance en région Nord-Pas-de-Calais, en Franche-Comté et en Haute-Normandie.
} 
les politiques de la nature (Pinton et al., 2007; Alphandéry et Fortier, 2007).

Dans un premier temps, nous montrerons le rôle déterminant joué par les associations naturalistes dans la production de données. L'examen approfondi du fonctionnement de l'une d'elle permettra de rendre compte de la manière dont s'opèrent, $d^{\prime}$ 'une part, la production de connaissances dans le cadre associatif et, d'autre part, les formes d'engagement bénévoles. Nous traiterons ensuite du processus de rationalisation des données en le resituant dans une histoire, marquée par des éléments de continuité et de rupture, pour aboutir à l'institutionnalisation de normes à travers le SINP. Enfin, nous montrerons comment ce processus de standardisation des données coïncide avec le mouvement de professionnalisation des associations naturalistes et concourt à modifier profondément le rôle et la place dévolus aux bénévoles et, plus largement, aux associations.

\section{Les associations naturalistes, principales pourvoyeuses de données}

La donnée naturaliste à laquelle il est fait référence ici est le produit d'une observation faite sur le terrain, comportant au minimum quatre types d'indications : la nature du taxon, le lieu, la date et le nom de l'observateur. En France, les données naturalistes disponibles sont majoritairement produites par les associations naturalistes. Héritières des sociétés savantes du XIXe siècle (Matagne, 1999), période où la science ne se distinguait pas de la protection de la nature (Raffin et Ricou, 1985), ces structures associatives ont vu le jour avec l'arrivée des scientifiques universitaires. Le recul de la systématique à l'Université dans le courant des années 1970, au profit de la biologie moléculaire, a laissé le champ libre aux associations (Maurin, 1997). Schématiquement, celles-ci se caractérisent par leur intérêt pour la production de connaissances, une légitimité en matière d'expertise et leur capacité à mobiliser des réseaux de bénévoles, amateurs pour la plupart (Micoud, 2001). L'amateur est dépeint ici " comme un citoyen ordinaire doté de compétences naturalistes qu'il développe et met au service d'une cause publique hors cadre professionnel, le plus souvent au sein d'une association » (Dupré et Micoud, 2007, p. 221). Il recouvre en réalité des profils différenciés qui se situent sur un continuum allant de $l^{\prime}$ «amateur du dimanche», dont l'expérience est limitée, la pratique irrégulière et le savoir fréquemment contesté, à l'amateur «éclairé » ou «chevronné», dont les compétences sont à rapprocher de celles des professionnels qui exercent leur activité dans le cadre d'un métier.

Parce qu'il y a dans les associations des gens prêts à livrer à peu de frais des données collectées par passion, les moyens alloués par les pouvoirs publics restent limités. Ainsi, de nombreux membres d'associations ont
Encadré 2. La Société mycologique du Nord de la France (SMNF)

Fondée en 1967, la SMNF, basée à la faculté des sciences de pharmacie et de biologie de Lille, se définit comme une association scientifique régionale, à but non lucratif, qui regroupe des bénévoles "s'intéressant aux champignons pour les reconnaître dans la nature, se familiariser avec leurs propriétés, leur classification, leur rôle, leur protection et leur éventuelle comestibilité ». Parmi les 220 adhérents, quelques dizaines seulement participent régulièrement aux manifestations organisées par l'association : sorties sur le terrain, conférences, séances d'initiation à la microscopie, session annuelle de formation, expositions mycologiques ouvertes au public, publications dans le bulletin de la société. À travers la collecte de données de terrain, ils concourent à l'inventaire de la fonge régionale. Ce projet a été mis en œuvre en 1991, à l'initiative du président de l'association, professeur et mycologue professionnel à la faculté de pharmacie, par ailleurs président de la Société mycologique de France. Les mycologues impliqués dans la réalisation de cet inventaire sont pour l'essentiel des amateurs couvrant toute la gamme des profils existants - du débutant à l'amateur éclairé puis chevronné -, encadrés de trois professionnels, universitaires, dont le président de l'association. Ce sont des personnes de sexe masculin dans leur grande majorité, plutôt âgées et issues de différents milieux sociaux. L'adhésion à une association mycologique représente un moyen privilégié d'apprentissage dans une spécialité réputée plus complexe que la botanique ou l'ornithologie.

le sentiment que leur travail de production de données naturalistes n'est pas reconnu par l'État à sa juste valeur. Cela explique l'enjeu majeur que représente la mise à disposition de l'information dans l'univers naturaliste. Si l'on adopte un point de vue utilitariste, la réticence à livrer les données produites trouve sa source dans la volonté d'exercer une forme de pouvoir et de monnayer des informations souvent considérées comme « un trésor de guerre » (Durand-Bourlier, 2003). A contrario, la production de connaissances ne saurait être envisagée uniquement en termes de ressource monnayable, mais elle peut être conçue, dans cet univers composé essentiellement de bénévoles, comme une richesse sur le plan du développement personnel et une activité associant des personnes au service du bien commun (Ellis et al., 2005). Afin de comprendre la manière dont coexistent ces différentes dimensions, nous nous sommes penchés sur le fonctionnement d'une association naturaliste, la Société mycologique du Nord de la France (SMNF), avec le souci d'identifier les formes d'engagement de ses adhérents (Encadré 2).

\section{Passions mycologiques}

La mycologie, à l'image des autres sciences naturalistes, a pour caractéristique de s'appuyer sur l'observation in situ. Elle fait ainsi partie des sciences de plein 
air (Callon et al., 2001). L'observation des champignons dans leur milieu naturel fait appel à des connaissances théoriques mais mobilise également d'importants savoirs pratiques. «Beaucoup de choses sont dans les bouquins - constate un professionnel de la SMNF -, mais y a un truc qui n'est pas dans les bouquins, c'est l'intuition. Les descriptions, les clefs de détermination ont beau être très détaillées, quelquefois les différences qui sont mentionnées ont l'air très superficielles comme ça; en fait, elles correspondent à des conditions de terrain qu'il n'est pas facile de transmettre par écrit. » Le savoir-faire des mycologues repose en effet sur la faculté à mobiliser certains sens, comme le regard ou l'odorat. Dans les entretiens, il est souvent fait allusion à tel ou tel mycologue, amateur ou professionnel, doté $\mathrm{d}^{\prime}$ " une mémoire invraisemblable des odeurs, des goûts, de l'allure du champignon » ou «d'un sens de l'observation étonnant, un coup d'œil, un nez extraordinaire». Cet intérêt commun pour le travail de terrain explique la frontière souvent ténue entre amateurs éclairés et professionnels, ainsi que les discours fréquents et animés qu'elle suscite (Delaporte, 1984 ; Rémy, 1995).

La production de savoirs, qui constitue une grande partie de l'action de la SMNF, est indissociable d'une dynamique collective. Les différentes manifestations, expositions, sorties sur le terrain, sessions de formation sont autant de cadres d'interaction pour les mycologues, qu'ils soient amateurs plus ou moins avertis ou professionnels, destinés à affiner les connaissances de chacun. "Au niveau de la SMNF - déclare le président-, on essaie de faire avancer la réflexion ou la motivation des gens sans forcément la détourner de leur motivation première qui est souvent respectable -, mais de les enrichir dans leur approche par cet aspect travail collectif, rigueur dans la détermination, prise en compte des champignons dans leur environnement [...]. Chacun fait en fonction de ses objectifs et du temps qu'il a à consacrer. » En tant que lieux d'apprentissage, ces collectifs sont aussi l'occasion fournie à chaque mycologue d'afficher ses compétences et d'en évaluer les limites. Ils fonctionnent ainsi comme une mise à l'épreuve de leur savoir par le terrain et par les autres. L'affiliation à l'association va souvent de pair avec la quête de reconnaissance, que celle-ci s'exerce au sein du groupe, par le biais des pairs, ou sur des scènes extérieures (voisinage, professionnel, etc.).

La production de connaissance est, en effet, indissociable des liens de sociabilité qui existent au sein du collectif. "Une association de bénévoles - fait remarquer un responsable d'association -, ce sont des gens qui se connaissent, des amis, des copains. On se voit, on discute, on s'engueule, donc il y a un aspect subjectif qui n'est pas scientifique mais qui est fondamental. » Pour beaucoup d'amateurs bénévoles affiliés à la SMNF, la mycologie est avant tout une activité où les rapports de convivialité, le sentiment d'appartenance au groupe constituent autant d'éléments déterminants dans l'investissement et le temps consentis à cette activité. Cet ensemble de paramètres : le désir de connaissances, le plaisir d'être ensemble, la quête de reconnaissance, mais aussi la mobilisation autour d'un projet commun, en l'occurrence l'inventaire de la faune à l'échelle régionale, participent à cette dynamique collective de production des savoirs au sein de l'association. Sans le travail des bénévoles qui ne comptent ni leur temps ni leur argent, les listes d'espèces fongiques régionales ne pourraient voir le jour. Réciproquement, ces inventaires sont une opportunité pour les amateurs d'enrichir leurs savoirs au contact des professionnels qui font montre d'une grande disponibilité aussi bien à l'égard des amateurs confirmés que des débutants. Cependant, la réalisation d'un tel projet repose largement sur la confiance et la capacité du président à exercer un rôle de médiation entre le monde des gestionnaires et des scientifiques, d'une part, et celui des amateurs bénévoles d'autre part. L'un d'eux (amateur éclairé) faisait remarquer :

«Ces inventaires biologiques sont devenus aussi l'alimentaire pour beaucoup de professionnels. Ça permet de récupérer des crédits [...]. Mais c'est dit, ce n'est pas caché. On ne prend pas non plus l'amateur pour le gogo qu'on piège dans un système. Ici, en tout cas, c'est très clair. »

Après avoir constaté combien la production de données par les bénévoles est liée aux rapports humains et aux valeurs qui les structurent, nous allons à présent montrer que le développement de ces inventaires et le processus de rationalisation des données qui l'accompagne s'inscrivent dans une histoire qui débute au cours des années 1970.

\section{Genèse du processus de rationalisation}

Depuis trois décennies, les données naturalistes sont de plus en plus mobilisées par les opérations de protection et de gestion de la nature et cette dynamique s'est traduite par une succession d'initiatives des pouvoirs publics pour rationaliser le dispositif de recueil et de traitement de l'information. La loi du 10 juillet 1976 joue un rôle fondateur dans la politique française de protection de la nature, en particulier par son article 1, qui stipule $q^{\prime}$ ' « il est du devoir de chacun de veiller à la sauvegarde du patrimoine naturel dans lequel il vit ». En fixant pour objectif de confectionner les listes rouges d'espèces menacées à protéger, la loi fournit un premier cadre de rationalisation de la collecte des données. Jusqu'à la fin des années 1980 prévaut, en effet, une conception de l'écologie qui considère les activités humaines comme des sources de perturbation des écosystèmes. Dans ce contexte, les associations se voient octroyer un agrément du ministère de l'Environnement qui leur donne une existence institutionnelle et leur permet de faire valoir leurs 
compétences (Lascoumes, 1994), notamment en matière de production de données. Les associations naturalistes s'investissent volontiers dans le suivi d'espèces rares, emblématiques ou patrimoniales dont la visibilité est censée susciter le soutien du public profane. La loi de 1976 consacre ainsi la priorité aux espaces et espèces protégés et entérine le «grand partage » entre nature « ordinaire» et nature « remarquable» qui pèse encore lourdement sur la structure des données naturalistes. L'organisation de l'inventaire des zones naturelles d'intérêt écologique faunistique et floristique (ZNIEFF), confiée en 1979 au Muséum national d'histoire naturelle (MNHN), confirme la priorité donnée par l'État aux espaces et espèces menacés. Sa réalisation a mobilisé un nombre très important de personnes ou d'institutions, souvent bénévoles et associatives, et suscité de nombreux problèmes méthodologiques. Le caractère peu ordonné de la construction de cet inventaire ne tient pas seulement à sa dimension expérimentale et à la faiblesse de ses moyens financiers. Il est aussi lié à la diversité des réseaux de connaissances présents dans chaque région, à la variété des configurations institutionnelles et à la qualité des rapports humains. Ces dimensions politiques et sociales jouent en effet un rôle important dans les ajustements successifs qui jalonnent le processus de production des données dans le cadre de l'inventaire (Rémy et al., 1999). Cette nouvelle phase de rationalisation a permis d'obtenir, grâce à la mobilisation des réseaux associatifs, un socle de connaissances à l'échelle de la France.

Au cours des années 1990, des changements profonds affectent les conceptions scientifiques dominantes, avec l'émergence de la notion de biodiversité. Reconnue pour ses «valeurs utilitaires » lors de la conférence de Rio de 1992 (Blandin, 2009), la conservation du vivant devient une norme pour l'action (Pinton et al., 2007). Elle s'appuie sur la prise en compte des différents niveaux d'organisation du vivant, dans une perspective non plus statique mais dynamique. La frontière entre espaces remarquables et nature ordinaire devient poreuse. Désormais, les activités humaines ne sont plus considérées comme une entrave à la biodiversité mais participent, pour certaines d'entre elles, a son maintien ; la politique de protection de la nature se transforme en une pratique gestionnaire et concerne tous les usagers de l'espace. La construction du réseau Natura 2000, qui incarne cette nouvelle préoccupation, et la diffusion des connaissances en écologie et dans les sciences de la conservation ont contribué à la prise en compte de multiples formes de connaissances - scientifiques, techniques, pratiques ou ordinaires - davantage tournées vers l'action. Plus généralement, l'approche en termes de conservation de la biodiversité porte avec elle l'idée de coproduction des savoirs et déplace le regard vers les espaces publics où les collectifs humains débattent des choix techniques et politiques nécessaires à l'élaboration des mesures de gestion.
Nos travaux menés sur l'élaboration concertée des plans de gestion des sites Natura 2000 ont montré que les associations ont été très sollicitées pour alimenter le travail de production de connaissances naturalistes. La reconnaissance de leur capacité d'expertise n'a toutefois pas modifié les réseaux existants, qui sont restés fragmentés et cloisonnés au regard des réseaux de compétences d'autres acteurs comme ceux de la forêt, de l'agriculture ou de la chasse (Pinton et al., 2007).

À l'issue de ce passage de la protection de la nature à sa conservation, le processus de rationalisation de la production des données naturalistes apparaît comme étant de plus en plus complexe. Il intègre des facteurs scientifiques, politiques et sociaux tels que l'évolution de la science en matière d'écologie, l'influence des conventions internationales, les rapports de force entre acteurs, les formes des politiques publiques de la nature, etc. On rejoint ainsi l'analyse développée par Lorimer (2006, p. 542): "We cannot understand biodiversity outside of the assemblage of people, practices, technologies and other nonhumans through which it is realized to us. " Dans cet assemblage, on peut identifier deux conceptions du savoir. L'une, au nom de la science, de sa traduction en action de gestion de la nature, de l'excellence professionnelle, concerne les méthodes de plus en plus technicisées de recueil, de traitement, de mutualisation et de mise à disposition des données. L'autre, issue d'une conception pluraliste de la production des connaissances, porte l'idée qu'il faut associer d'autres acteurs que les naturalistes, scientifiques et amateurs, à la production de données. Dans les faits, ces deux conceptions sont complémentaires et susceptibles d'être mêlées, même si, comme on va le voir avec le SINP, la priorité est souvent donnée à la dimension instrumentale de la rationalité.

\section{Le SINP ou l'institutionnalisation de normes liées à la production des données}

La construction du SINP (Encadré 1) représente une nouvelle étape dans le processus de rationalisation de la production des données naturalistes en France. Le dispositif conçu par le ministère comporte une dimension partenariale incarnée par un comité national ${ }^{5}$, tout en présentant un aspect prescriptif. Ce dernier consiste, au nom de la rationalité scientifique, à définir des référentiels et des méthodologies communs ainsi que des critères

\footnotetext{
${ }^{5}$ Celui-ci est composé d'une cinquantaine de membres parmi lesquels figurent les principales structures impliquées dans la production, la validation, la gestion et la valorisation des données à l'échelle de l'Hexagone : collectivités locales, établissements de recherche et universités, organisations non gouvernementales et usagers comprenant notamment les chasseurs et les pêcheurs, établissements publics et opérateurs gestionnaires, administrations. Ce comité a une visée essentiellement consultative.
} 
permettant d'attester de la qualité des données. Une «coordination scientifique » pilotée par le MNHN est chargée d'élaborer ces normes. Il revient ensuite aux régions de mettre en place une organisation qui facilite les échanges, favorise la mutualisation et l'accès aux données, sous réserve qu'elles se conforment aux normes établies à l'échelon national. Ce dernier point va animer une partie des débats du comité national du SINP, en particulier au cours de la rédaction du protocole, dont la première version stipule qu'il est délicat de garantir aux données naturalistes un «fondement scientifique leur attribuant une réelle légitimité permettant ainsi de contribuer au débat public et d'éviter le contentieux ${ }^{6} »$. Ce qui revient à réitérer les accusations portées depuis longtemps sur la «mauvaise » qualité du travail des associations naturalistes. Lors de la réunion du 15 avril 2006 du comité national, le représentant de France Nature Environnement (FNE) en appelle à "rétablir une confiance avec la communauté naturaliste nationale » d'où proviennent les données, certes de qualité inégale, reconnaît-il, mais qui approvisionnent les divers systèmes. En retour, la volonté du MNHN de contrôler l'ensemble du processus est critiquée par plusieurs intervenants. Le représentant de la Fédération des conservatoires botaniques nationaux tient à rappeler le rôle officiel joué par sa structure en matière de production de données et d'expertise auprès des pouvoirs publics et insiste sur la nécessité d'impliquer l'ensemble des acteurs ayant reçu une mission de service public. Il est aussi implicitement reproché au MNHN d'avoir violé les engagements de confidentialité et d'avoir mis en ligne des données fournies par les associations sans les en avoir averties. Ainsi, la question des instances légitimes a-t-elle été posée avec insistance, notamment la mission de coordination scientifique confiée au MNHN. De la même manière, la nécessité d'impliquer les associations tout au long du processus a été affirmée ${ }^{7}$.

L'avènement du SINP et les initiatives régionales qui l'ont précédé, notamment en région Nord-Pas-de-Calais, ont conduit à faire coexister des conceptions différentes de la donnée. L'une, incarnée par le SINP, tend à faire de la donnée un «morceau » de nature objectivée, défini sous une forme conventionnelle, aisément mobilisable à travers des bases de données. L'autre, portée par un amateur éclairé, membre d'une association ornithologique de la région Nord-Pas-de-Calais, pour qui la donnée est multiforme et inscrite dans une histoire et un contexte :

« La donnée brute mise dans un système... entre les mains de personnes inconnues, moi, je m’en méfie beaucoup. Par

6 Le texte du projet de protocole est disponible sur le portail du SINP : http://www.naturefrance.fr/spip.php?article5 (« Projet du protocole transmis avec l'invitation » à la réunion du 25 janvier 2007 du comité national).

7 Le compte rendu de la réunion du comité national est disponible sur le portail du SINP : http://www.naturefrance.fr/spip. php?article6 (CR validé réunion du 5 avril 2006). exemple, vous vous baladez, vous voyez un couple de chouettes. C'est une donnée : tel endroit, telle heure [...]. Vous allez mettre ça dans une base de données. Comment interpréter ça ? Ce sont deux adultes? Est-ce vraiment un couple? Y en a une qui est dans un trou : est-ce qu'elles vont nicher? Vous voyez, y a beaucoup d'interprétations possibles. Si vous êtes ornithologue, que vous connaissez le coin et que vous savez que la chouette chevêche niche là depuis dix, quinze ans, vous allez vous dire: couple nicheur de chouettes chevêches comme traditionnellement dans le secteur. Si vous connaissez le coin et que c'est la première fois que le couple arrive, ça a tout à fait une autre signification. C'est-à-dire qu'il y a un nouveau territoire qui vient d'être conquis par un couple de chouettes chevêches, espèce en fort déclin. Alors, qu'est-ce que ça veut dire? Est-ce qu'elle n'est plus en déclin ${ }^{8} ? .$. »

À travers ce récit, notre interlocuteur met l'accent sur le travail d'interprétation des données naturalistes, qu'il juge «difficile [comparé à celui] des scientifiques qui travaillent en laboratoire avec des protocoles très précis ». Données que les associations sont désormais tenues, conformément à la convention d'Aarhus, de mettre à la portée du plus grand nombre. Et de conclure : «Les données de l'association sont nos données. Nous les publions nous-mêmes et nous les analysons nous-mêmes, car nous sommes les seuls à pouvoir les analyser convenablement. » L'existence de conceptions différentes de la donnée se manifeste également à travers le mouvement de restructuration qui s'effectue au sein des associations naturalistes.

\section{Les associations, entre bénévolat et professionnalisation}

Dans le courant des années 1990, les plus grandes associations naturalistes connaissent des transformations allant dans le sens d'une professionnalisation de leur activité (Micoud, 2001). Cette notion omniprésente, qui incarne les formes actuelles de légitimation et d'institutionnalisation, est toutefois peu définie, sinon empiriquement. On entend souvent par professionnalisation le processus d'extension du nombre de personnes exerçant leur métier au sein des associations. Mais on doit aussi associer à cette notion d'autres composantes : des compétences naturalistes reconnues (maîtrise des outils scientifiques, capacités d'expertise), une gestion rigoureuse de l'association (conditions d'embauche codifiées, comptabilité suivie, moyens adéquats de formation), l'existence de partenariats (avec les collectivités locales, le privé ou l'Union européenne) et l'appartenance à des réseaux. Cette évolution doit être rapportée à la conjonction de plusieurs phénomènes. Avec la multiplication des politiques de conservation de la biodiversité, on assiste à une

\footnotetext{
8 Entretien, mai 2006.
} 
remise en cause du quasi-monopole de l'expertise des associations au profit de bureaux d'études spécialisés, faisant appel à des salariés qualifiés. Ce phénomène intervient dans un contexte marqué par une perte de confiance relative des pouvoirs publics vis-à-vis du monde associatif, accusé de produire des données peu fiables, et par une diminution constante des financements qui leur sont alloués ${ }^{9}$. La professionnalisation apparaît alors, aux yeux d'une fraction des associations, comme un moyen de reconquérir une légitimité tout en permettant de développer des activités marchandes de substitution. De façon concomitante, le développement d'une offre de formation dans le domaine de l'environnement, à compter des années 1980-1990, assuré par des institutions publiques (universités) ou privées, mais aussi des organismes spécialisés comme l'Atelier technique des espaces naturels (ATEN), a contribué à donner corps à ce mouvement de professionnalisation. Celui-ci est cependant loin d'être uniforme. Il prend des formes diverses que l'on peut situer sur un continuum allant des associations qui continuent à recourir à un fonctionnement basé sur le bénévolat à celles qui privilégient le recours à des salariés permanents qualifiés. Entre ces deux pôles existe toute une gamme de situations intermédiaires. Certains membres d'associations reconnus comme experts sont mandatés par des bureaux d'études pour effectuer des travaux ponctuels. Des associations s'attachent les services de permanents tout en s'appuyant sur un réseau de bénévoles. D'autres, enfin, comme ODONAT, s'efforcent de favoriser la mise en réseau des structures détentrices de données au sein du monde associatif.

Dans le contexte actuel de professionnalisation des structures et de rationalisation de la production des données, toute la difficulté pour les associations naturalistes, qui mêlent dans des proportions plus ou moins fortes bénévoles et salariés, activités non marchandes et marchandes, consiste à maintenir l'équilibre entre, d'un côté, ce qui fait la raison d'être de l'association et, de l'autre, les moyens qu'elle met en œuvre pour assurer son fonctionnement (Enjolras, 1998; Laville et al., 2001). Le développement $\mathrm{d}$ 'une rationalité technico-instrumentale qui accompagnel'entrée en économie des associations, même si celle-ci demeure limitée, pose en effet la question du sens de la participation bénévole. Si, comme le suggère Prouteau (2003), le bénévolat demeure un engagement au service d'une cause, il devient dans ce nouveau contexte une ressource en main-d'œuvre dans un processus de production générateur de tensions internes. Trois exemples permettent d'illustrer ces tensions. La Ligue pour la protection des oiseaux de Franche-Comté, forte de 250 membres et proche de l'Université, a développé, à la fin des an-

\footnotetext{
9 Le montant des aides de l'État accordées aux associations de protection de la nature et de l'environnement a chuté de près de $33 \%$ entre 2002 et 2005 (cf. http://www.associations. regioncentre.fr/home/sujet_du_mois.php?id_sujet=28\&impri).
}

nées 1990, une activité professionnelle en embauchant sept salariés qualifiés. Tout en arguant de la nécessité de s'engager dans une expertise de plus en plus pointue pour répondre aux sollicitations multiples (Natura 2000, ZNIEFF nouvelle génération), son responsable se défend d'aller à l'encontre de la logique associative : «Nous, on n'est pas des producteurs de données, on est une société d'étude et de protection de la nature. Les données, c'est un outil ; notre finalité, ce n'est pas de produire des données $^{10}$.» Dans un registre un peu différent, le discours des responsables du Groupe ornithologique Nord $(\mathrm{GON})$, confrontés au projet de mise en place d'un réseau destiné à structurer la donnée naturaliste dans le NordPas-de-Calais, souligne avec une plus grande acuité les tensions suscitées par ce projet :
« Le GON adhère à l'idée d'une convention commune où les degrés de diffusion [des données] seraient clairement énoncés. Avant toute diffusion, une consultation du conseil d'administration sera nécessaire. Cependant, il est défini que le GON doit continuer à faire vivre sa base [de données] et à satisfaire les demandes des adhérents ; la diffusion large ou la cartographie ne saurait être dans leurs finalités et dans leurs moyens ${ }^{11}$.»

Et les auteurs de cette note de souligner «la répugnance passée [du GON] à l'égard d'une quelconque utilisation des données » et la tendance de ses responsables à concevoir le travail de collecte et de relevé dans une optique de "plaisir». Enfin, l'initiative lancée par ODONAT et reprise par FNE est justifiée par l'un des responsables en ces termes:

«Les associations revendiquent aujourd'hui d'être gestionnaires et propriétaires de la majorité ou d'un très grand nombre de données naturalistes en France, de façon historique. Nous n'avons pas accumulé ces connaissances pour les garder dans des armoires et qu'elles ne servent à personne! L'objectif même des associations, c'est le porté à connaissance des décideurs pour que ceux-ci puissent appliquer des politiques publiques de protection de la nature $»$.

À condition, poursuit notre interlocuteur, que

\begin{abstract}
« les données ne soient pas utilisées à n'importe quelle fin et que l'on se donne les moyens, à travers la mise en place d'un code de déontologie, d'éviter les phénomènes de pillage par les bureaux d'études qui reprennent les données sans jamais citer l'association ni l'observateur [... ] ou les erreurs d'interprétation des données ».
\end{abstract}

À travers cette initiative, FNE exprime ses réticences à privilégier une rationalisation de type instrumental, fondée sur la standardisation et la logique industrielle au détriment du bénévolat qui met en avant la confiance, les relations de face-à-face, la réciprocité et l'ancrage territorial.

\footnotetext{
10 Entretien, mars 2005.

11 Extrait d'un document de travail réalisé par la DIREN NordPas-de-Calais.
} 


\section{Conclusion}

L'adoption du SINP par le MEDAD marque une nouvelle étape dans le processus de rationalisation des données sur la nature en France. S'il est encore trop tôt pour évaluer l'impact d'un tel projet, le débat public dont il est l'objet voit se manifester les réticences d'une partie significative des associations naturalistes, qui perçoivent cette initiative comme une forme d'instrumentalisation. Aux yeux des responsables que nous avons rencontrés, la production de données ne saurait être une fin en soi. L'engagement bénévole dans les associations relève, en effet, de motifs variés qui s'inscrivent dans un continuum entre des intérêts personnels (curiosité, passion, désir de connaissance, volonté d'affiliation à un groupe, reconnaissance par les pairs, etc.) et un sentiment de responsabilité à l'égard de la nature. Le SINP agit, de fait, comme un révélateur de l'existence de conceptions différentes de la donnée : d'une part, celle qui s'inscrit dans des rapports de type personnalisé fondés sur la réputation des collecteurs et notamment leur expérience de terrain; d'autre part, celle qui conçoit la donnée comme une ressource facilement mobilisable et nécessitant un travail d'homogénéisation préalable.

Le processus de normalisation porté par le SINP, souvent associé par ses promoteurs à des valeurs fondées sur l'efficacité, la transparence, la conservation du vivant, la démocratie - tout citoyen est désormais en droit de pouvoir accéder à l'information -, n'est toutefois pas sans contreparties. La standardisation des données conduit à extraire celles-ci de leur contexte social et environnemental et donc à se priver d'un certain nombre de variables souvent essentielles dans l'usage et l'interprétation de cette information. Elle conduit en outre à privilégier les savoirs d'inventaire plus facilement quantifiables (Bowker, 2000) et à laisser dans l'ombre d'autres formes de connaissances plus complexes et difficiles à mobiliser, comme les savoirs gestionnaires ou les savoirs pratiques. $C^{\prime}$ est en tout cas la tendance observée au sein du SINP, et dont il importera de vérifier si elle se confirme dans les années à venir. Enfin, en choisissant d'allouer en priorité des moyens financiers aux associations engagées dans la voie de la professionnalisation, le ministère en charge de l'environnement ne risque-t-il pas de se priver des apports d'une partie d'entre elles? Dans un domaine aussi vaste et complexe que celui de la biodiversité, le ministère peut-il raisonnablement se passer du travail fourni par un grand nombre de bénévoles ou encore par d'autres catégories d'acteurs, comme les chasseurs, qui disposent de moyens financiers importants pour assurer la collecte des données (Alphandéry et Fortier, 2007)?

La professionnalisation du secteur associatif et le mouvement de standardisation des données entrepris dans le cadre du SINP concourent à introduire une division des tâches entre production et traitement de la donnée et, dans le même temps, à renforcer le clivage entre amateurs et professionnels. Les bénévoles, qui représentent la majorité des effectifs au sein des associations, résistent à cette nouvelle organisation du savoir sur la nature précisément parce qu'elle tend à dessaisir un certain nombre d'entre eux de leur capacité d'expertise et à les reléguer au rang de simples pourvoyeurs de données. Ce type d'expertise relève en effet de plus en plus d'un travail de compilation et d'interprétation d'informations qui suppose des compétences et la maîtrise d'outils souvent très sophistiqués exigeant de faire appel à des professionnels. Cette délégation de l'expertise aux professionnels apparaît pour le moins paradoxale dans un contexte où les besoins de connaissance sont croissants. Par ailleurs, ce double mouvement de professionnalisation et de standardisation traduit une évolution dans les rapports entre le ministère en charge de l'environnement et les associations. La loi de 1976 avait intégré celles-ci à la politique de protection de la nature en leur confiant des missions de services publics, les conviant ainsi à défendre le bien commun contre les atteintes au patrimoine naturel. À travers la tendance qui consiste à faire des associations des prestataires de services et à les cantonner dans un rôle d'expertise, le ministère ne semble plus attendre d'elles qu'elles soient porteuses d'une capacité d'initiative politique. Si cette tendance se confirmait, la conception d'une gestion écologique tournée exclusivement vers des préoccupations scientifiques et techniques (Alphandéry et al., 1991) participerait, conformément à ce qu'écrivait Lascoumes en 1994, au renforcement du pouvoir des experts, aboutissant à laisser les «écocrates » penser et gérer le vivant. Paradoxe étonnant si l'on considère qu'à ses débuts, la pensée écologique critiquait «la domination du monde par la raison technique » (Lascoumes, 1994) et tenait la diversité des structures associatives et leur autonomie comme une condition de la démocratie.

\section{Références}

Alphandéry, P., Bitoun, P., Dupont, Y., 1991, L'Équivoque écologique, Paris, La Découverte.

Alphandéry, P., Fortier, A., 2007, A new approach to wildlife management in France. The formulation of the ORGFSH as tools for the conservation of biodiversity, Sociologia Ruralis, $47,1,42-62$.

Balmford, A., Bennun, L., ten Brink, B., Cooper, D., Côté, I.M., Crane, P., Dobson, A., Dudley, N., Dutton, I., Green, R.E., Gregory, R.D., Harrison, J., Kennedy, E.T., Kremen, C., Leader-Williams, N., Lovejoy, T.E., Mace, G., May, R., Mayaux, P., Morling, P., Phillips, J., Redford, K., Ricketts, T.H., Rodriguez, J.P., Sanjayan, M., Schei, P.J., van Jaarsveld, A.S., Walther, B.A., 2005. Science and the Convention on Biological Diversity's 2010 target, Science, 307, 5707, 212213.

Blandin, P., 2009. De la protection de la nature au pilotage de la biodiversité, Versailles, Quae. 
Boussard, V., Maugeri, S., 2003. Introduction, in Boussard, V., Maugeri, S. (Eds), Du politique dans les organisations : sociologies des dispositifs de gestion, Paris, L'Harmattan, 25-61.

Bowker, G.C., 2000. Biodiversity datadiversity, Social Studies of Science, 30, 5, 643-683.

Callon, M., Lascoumes, P., Barthes, Y., 2001. Agir dans un monde incertain, Paris, Le Seuil.

Charvolin, F., 2009. Des sciences citoyennes? La question de l'amateur dans les sciences naturalistes. Communication au colloque Sciences citoyennes et biodiversité, Tela Botanica, Montpellier, 22-23 décembre (www.tela-botanica. org/wikini/colloquescb/).

Delaporte, Y., 1984. Des insectes et des hommes, Les Temps modernes, 450, 1235-1263.

Dupré, L., Micoud, A., 2007. Savoirs publics sur la nature et politiques publiques de l'environnement : rôle et place des naturalistes amateurs et des professionnels, in Charvolin, F., Micoud, A., Nyhart, L.K., Des sciences citoyennes? La question de l'amateur dans les sciences naturalistes, La Tour d'Aigues, L'Aube, 219-232.

Durand-Bourlier, 2003. Quels réseaux d'information pour une meilleure prise en compte de la biodiversité dans les politiques publiques? Rapport pour le conseil régional du Nord-Pasde-Calais.

Ellis, R., Grove-White, R., Vogel, J., Waterton, C., 2005. Nature: Who Knows?, [s.1.], English Nature / Lancaster University / Natural History Museum.

Enjolras, B., 1998. Crise de l'État-providence, lien social et associations : éléments pour une socio-économie critique, Une seule solution, l'association? Socio-économie du fait associatif, Revue du MAUSS semestrielle, 11, 223-236.

Lascoumes, P., 1994. L'Éco-pouvoir, Paris, La Découverte.

Laville, J-L., Caillé, A., Chanial, P., Dacheux, É., Eme, B., Latouche, S., 2001. Associations, démocratie et société civile, Paris, La Découverte/MAUSS/CRIDA.

Lorimer, J., 2006. What about the nematodes? Taxonomic partialities in the scope of UK biodiversity conservation, Social and Cultural Geography, 7, 4, 539-558.

Reçu le 31 août 2009. Accepté le 1er décembre 2010.
Matagne, P., 1999. Aux origines de l'écologie, Paris, Éditions du CTHS.

Maurin, H., 1997. Les missions du service du patrimoine naturel (IEGB/MNHN) : connaissance et suivi du patrimoine naturel, espèces et espaces. Communication au colloque Espaces naturels terrestres : de la décision à l'action, AICEF, Orléans, octobre (supplément $\mathrm{n}^{\circ} 35$ de Forestier).

Micoud, A., 2001. La nébuleuse associative, in Rochefort, R., Voynet, D. (Eds), L'Environnement, question sociale : dix ans de recherche pour le ministère de l'Envionnement, Paris, Odile Jacob, 119-129.

Ministère de l'Écologie et du Développement durable, 2004. Stratégie nationale pour la biodiversité : enjeux, finalités, orientations.

Mougenot, C., 2003. Prendre soin de la nature ordinaire, Paris, MSH/INRA.

Pinton, F. (Ed.), Alphandéry, P., Billaud, J.-P., Deverre, C., Fortier, A., Gesniaux, G., 2007. La Construction du réseau Natura 2000 en France, Paris, La Documentation française.

Prouteau, L., 2003. Des associations sous tensions, in Prouteau, L. (Ed.), Les Associations : entre bénévolat et logique d'entreprise, Rennes, Presses Universitaires de Rennes, 7-26.

Raffin, J-P., Ricou, G., 1985. Le lien entre les scientifiques et les associations de protection de la nature : approche historique, in Cadoret, A. (Ed.), Protection de la nature : histoire et idéologie, de la nature à l'environnement. Colloque de Florac, 1985, Paris, L'Harmattan, 61-73.

Rémy, É., 1995. L'élaboration d'un savoir naturaliste, Études rurales, 137, 55-72.

Rémy, É. (Ed.), Alphandéry, P., Billaud, J-P., Bockel, N., Deverre, C., Fortier, A., Kalaora, B., Perrot N., Pinton, F., 1999. La Mise en directive de la nature : de la directive Habitats aux prémices du réseau Natura 2000. Rapport pour le ministère de l'Aménagement du territoire et de l'Environnement, Direction de l'évaluation environnementale et des études économiques, Paris.

Scholes, R.J., Mace, G.M., Turner, W., Geller, G.N., Jürgens, N., Larigauderie, A., Muchoney, D., Walther, B.A., Mooney, H.A., 2008. Ecology: Toward a global biodiversity observing system, Science, 321, 5892, 1044-1045. 